\title{
Phase Change Characteristics of Ultra-Thin Liquid Argon Film over different Flat Substrates at High Wall Superheat for Hydrophilic/Hydrophobic Wetting Condition: A Non-Equilibrium Molecular Dynamics Study
}

\begin{abstract}
Mohammad. NasimHasan", S. M. Shavik, K. F. Rabbi, K.M. Mukut, A. K. M. M. Morshed
Department of Mechanical Engineering, Bangladesh University of Engineering and Technology, BUET, Dhaka, 1000, Bangladesh, *Email: nasim@me.buet.ac.bd

Abstract:

Non-equilibrium molecular dynamics simulations have been conducted to understand the effect of solid-liquid interfacial wettability and surface material on the phase change phenomena of the thin liquid argon film placed over flat substrate at high wall superheat. The molecular system consists of a three phase simulation domain involving solid wall, liquid argon and argon vapor. After the system is thermally equilibrated at $90 \mathrm{~K}$ and kept in equilibrium for a while, a high wall superheat ( $250 \mathrm{~K}$ that is far above the critical temperature of argon) is induced at the liquid boundary so that the liquid undergoes ultrafast heating. Both hydrophilic and hydrophobic surfaces were considered in the present study in order to observe the effect of surface wettability on phase change characteristics for three different solid substrate materials namely, Platinum (Pt), Silver (Ag) and Aluminium (Al). Results obtained in the present study are discussed in terms of transient atomic distribution inside system domain, heat flux characteristics across the solid-liquid interface together with evaporative mass flux from liquid argon. Simulation results show that, depending on the surface wetting condition, the phase change process appears to be very different (explosive/ diffusive) for all three substrate materials under consideration. Among three materials considered herein, $\mathrm{Al}$ is found to offer the least favourable condition for phase change process while $\mathrm{Pt}$ and $\mathrm{Ag}$ show similar heat and mass transfer characteristics for both hydrophilic and hydrophobic wetting conditions. Surface wettability effect is found to be more prominent than the effect of substrate material in thin film liquid phase change phenomena.
\end{abstract}

Keywords: Wettability, molecular dynamics, evaporation, explosive boiling.

\section{Introduction}

Boiling heat transfer phenomena have been a topic of great interest in the field of Heat Transfer Research. In recent years, the phase transition of thin film liquid over a solid surface has predominantly received attention because of its practical and scientific applications in various emerging engineering fields such as, thermal management of micro or nano-electronic systems, laser surgery and laser steam cleaning of a solid surface, refrigeration, cryogenics, etc. In recent times, explosive boiling that is a very swift liquid to vapor phase transition resultingfrom ultrafast liquid heating (in whichthe liquid attains a very high superheat far above its saturation temperature)has attracted researcher's interest due to its applications and. Numerous experiments and numerical studies have been conducted recently to understand the behavior of the phase transition during evaporation and explosive boiling of the thin liquid film. Yet a lack of established theory for sufficiently predicting the characteristics, especially the rate of heat transfer during explosive boiling still makes this study a challenging one. Moreover, due to the small scale, performing experimentation is sometimes difficult. As a result, many researchers have tried to reveal the characteristics of these phenomena numerically, particularly in recent years by performing non-equilibrium molecular dynamics simulation (NEMD). Molecular dynamics simulation is becoming more and more prevalent in this field because of its ability to investigate such phenomena from the microscopic view point. In many molecular dynamics studies, researchers have given intensive focus on various issues of phase change process. Kimura and Maruyama [1] simulated heterogeneous nucleation of argon liquid droplet on a solid surface modeled as a constant temperature heat bath using phantom molecules. Sekine et al. [2] simulated liquid-vapor nucleation of Lennard-Jones fluid by molecular dynamics method and calculated the nucleation rate at three different temperatures by survival probability of the bubble-free state. Yu and Wang [3] simulated evaporation of thin argon liquid films and evaluated the net mass flux. Also they found a very thin nonevaporating layer and compared it with theoretical models. Nagayama et al. [4] carried out MD simulations to examine the bubble behavior confined in a nanochannel with emphasis on nucleation phenomenon. Maroo and Chung [5] performed molecular dynamics simulation of platinum heater and associated nano-scale liquid argon film evaporation and colloidal adsorption characteristics. Abovementioned studies are performed to particularly understand the behavior of the heterogeneous phase transition of thin liquid on a flat solid surface.

Many studies on explosive boiling has been conducted to confirm that the rate of heat transfer during the phase transition can be significantly increased if nanostructures are embedded over the flat solid surface. Morshed et al. [6] studied the effect of nanostructures 
on evaporation and explosive boiling of thin liquid films through molecular dynamics simulation considering cylindrical nanoposts. Seyf and Zhang [7] studied the effect of spherical nanostructure and confirmed the significant effect of size and shape of the nanostructures on explosive boiling. Along with the nanostructure, the study of the surface effects like change of solid-liquid surface wettability coupled with different solid surface material can add some new dimension to this kind of research. The solid surfaces are frequently termed as hydrophilic or hydrophobic on the basis of solid-liquid surface wettability, which is commonly defined by the contact angle between the liquid and solid surface. The nature of solid surface (hydrophilic or hydrophobic) has great effect on characteristics of evaporation and explosive boiling. Maruyama and Kimura [8] simulated a heterogeneous nucleation of argon vapor bubble on a solid Pt surface and measured the apparent contact angle. Hens et al. [9] performed MD simulation of liquid argon film to understand the mechanism of bubble nucleation with particular emphasis on surface texture and found that the boiling phenomena is considerably affected by the surface wettability.

In the present study, by using non-equilibrium molecular dynamics simulation (NEMD), phase change characteristics of thin liquid argon film over solid surface has been studied by considering different surface material and solid-liquid interfacial wettability for a high wall superheat. Three different solid materials have been considered which are Platinum $(\mathrm{Pt})$, silver (Ag) and aluminum (Al). Based on surface wettability two cases have been considered; hydrophilic and hydrophobic. The objective of this study is to find out the effect of both surface material and solid-liquid interfacial wettability on phase transition and other associated phenomena by closely monitoring the temperature, number density, heat transfer rate etc. during the phase change process.

\section{Simulation Method}

The simulation domain considered in this study contains liquid argon layers employed over a solid wall and argon vapor which resides over the liquid layers. The initial configuration of the simulation domain is shown in Fig. 1, where the simulation cell is a cuboid having a dimension of $7.35 \mathrm{~nm} \times 70.0 \mathrm{~nm} \times 7.35 \mathrm{~nm}(\mathrm{x}$ $\times \mathrm{y} \times \mathrm{z}$ ).At the bottom of the simulation box eight monolayers of solid $(\mathrm{Pt} / \mathrm{Ag} / \mathrm{Al})$ atoms are placed which are arranged in a FCC ( $\left.\begin{array}{lll}1 & 0 & 0\end{array}\right)$ lattice corresponding to their density. The solid wall has a height of $1.505 \mathrm{~nm}$. Different layers of the solid wall had different functions; the bottom layer of the wall was kept fixed; next two layers were set as the heat source and Langevin thermostat was applied to these layers of the solid wall and the remaining five layers at top were considered as the solid wall through which heat is transferred to the liquid argon layers. The liquid argon layer has a total thickness of $3.01 \mathrm{~nm}$ and placed over the solid wall corresponding to its density of $1.367 \times 10^{3}$ $\mathrm{kg} / \mathrm{m}^{3}$ at $90 \mathrm{~K}$. The rest of the space is filled with argon vapor atoms. The total number of atoms in the system varied for different cases as each solid material has different density as tabulated in Table-1.

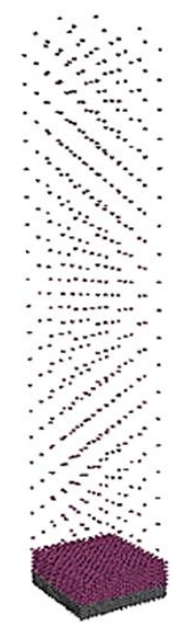

Fig. 1.Initial configuration of the simulation domain.

Table 1.Summary of the molecular system.

\begin{tabular}{c|c|ccc}
\hline \multirow{2}{*}{ Materials } & \multirow{2}{*}{$\begin{array}{c}\text { Density } \\
\mathrm{kg} / \mathrm{m}^{3}\end{array}$} & \multicolumn{3}{|c}{ No. of Atoms } \\
\cline { 3 - 5 } & 21450 & 5476 & 4193 & 9669 \\
$\mathrm{Pt}$ & 10500 & 5184 & 4193 & 9377 \\
$\mathrm{Ag}$ & 2700 & 5004 & 4193 & 9197 \\
$\mathrm{Al}$ & &
\end{tabular}

The intermolecular forces for all atoms within the simulation domain were determined by the well-known Lennard-Jones (LJ) 12-6 potential [10]:

$$
\phi(r)=4 \varepsilon\left[\left(\frac{\sigma}{r}\right)^{12}-\left(\frac{\sigma}{r}\right)^{6}\right]
$$

The values of energy parameter, $\varepsilon$ and length parameter, $\sigma$ for the liquid-liquid (Ar-Ar), solid-solid (Pt-Pt, Ag$\mathrm{Ag}$ and, $\mathrm{Al}-\mathrm{Al}$ ) interaction potentials are tabulated in table 2 . The energy parameter $\varepsilon_{\text {liquid-solid }}$ of solid-liquid interaction potential was varied for different cases to change the wettability of substrate surface based on the study of Hens et al. [9]. They showed that the contact angle of argon placed over flat solid surface changes when solid- liquid interaction potential is changed and as the surface wettability is mostly defined by the contact angle, the study suggested that, when $\varepsilon_{\text {liquid-liquid }}$ $<\varepsilon_{\text {liquid-solid }}$, the surface can be considered as hydrophilic and when $\varepsilon_{\text {liquid-liquid }}>\varepsilon_{\text {liquid-solid, }}$ surface can be considered as hydrophobic. Based on their study, two different types of surface wetting conditions have been assumed in the present study based on the surface. When $\varepsilon_{\text {liquid-liquid }} / \varepsilon_{\text {liquid-solid }}=0.5$, the surface is considered as hydrophilic, when $\varepsilon_{\text {liquid-liquid }} / \varepsilon_{\text {liquid-solid }}=$ 2 , surface is considered as hydrophobic. A cut off distance of $4 \sigma_{A r-A r}$ was used for the potential functions to increase the computational efficiency. 
Table 2.Lennard-Jones (LJ) potential parameters of different materials considered in thepresent study

\begin{tabular}{c|c|c}
\hline Interaction & $\boldsymbol{\sigma}(\boldsymbol{n m})$ & $\boldsymbol{\varepsilon}(\boldsymbol{e V})$ \\
\hline $\mathrm{Ar}-\mathrm{Ar}$ & 0.3400 & 0.0104 \\
$\mathrm{Pt}-\mathrm{Pt}$ & 0.2475 & 0.5200 \\
$\mathrm{Ag}-\mathrm{Ag}$ & 0.2574 & 0.3510 \\
$\mathrm{Al}-\mathrm{Al}$ & 0.2551 & 0.4080 \\
\hline
\end{tabular}

The equation of motion for each particle was integrated using velocity-Varlet algorithm with 5 fs time step. Periodic boundary conditions were applied in the $x$ and $z$ directions, whereas a simple non periodic fixed boundary condition is assumed in the $y$-direction with adiabatic and elastic boundary at the top i.e. the argon atoms are reflected back to the simulation domain from the top boundary without any change of momentum and kinetic energy.

The simulations of this study for all cases were carried out in three different stages. At the first stage, starting from the initial configuration of the simulation domain the entire system was set at a uniform temperature of 90 $\mathrm{K}$ by using Langevin thermostat. At this condition simulation was run for $1 \mathrm{~ns}$. At the second stage, the Langevin thermostat was switched off and the system was allowed to equilibrate for $1 \mathrm{~ns}$. To check whether the system was in the equilibrium state or not, the thermodynamic states i.e. temperature, energy, pressure and density of argon atoms were closely monitored during this period. At the equilibrium period, the temperature of the argon and solid wall fluctuated around $90 \mathrm{~K}$ for all the cases. Figure 2 shows the number density profile of argon on hydrophilic platinum surface during the equilibrium period. The number density profile is compared with the phase diagram of the Lenard Jones system [10] and previous studies [6-7], from the comparison, it can be said that the system is indeed in thermal equilibrium. For the silver and aluminum surface and hydrophobic case, the number density profiles of argon during equilibrium period show the similar characteristics as shown in Fig. 2. After the attainment of the equilibrium, to initiate the phase change process, the Langevin thermostat of solid wall $\left(T_{w}\right)$ was set to $250 \mathrm{~K}$. The upper three layers of the wall responded quickly and reached the target temperature within $50 \mathrm{ps}$. The simulation in this condition was run for $5 \mathrm{~ns}$.

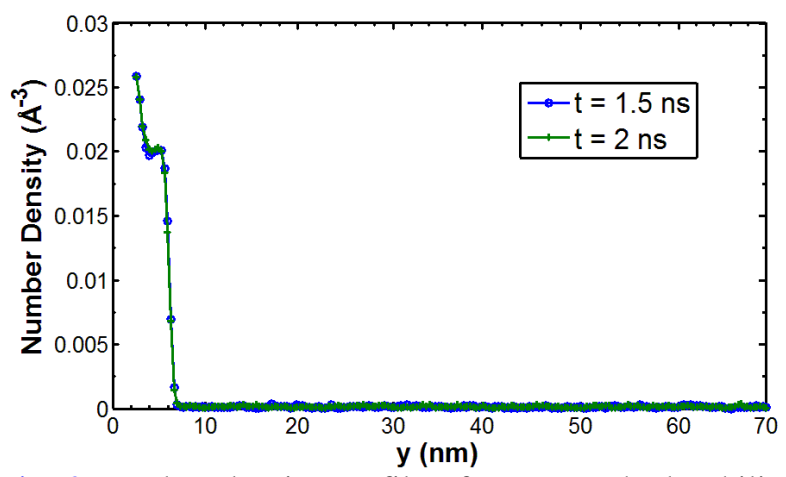

Fig. 2.Number density profile of argon on hydrophilic Pt-surface during equilibrium.
All the simulations of this study were performed using LAMMPS (Large scale Atomic/Molecular Massively Parallel Simulator) [11] which is an open source code for classical MD simulation developed by Sandia National Laboratory, USA and visualizations were done using VMD (Visual Molecular Dynamics) [12].

\section{Results and Discussion}

Understanding the phase change characteristics of thin film liquid argon at relatively higher wall superheat $\left(\mathrm{T}_{\mathrm{w}}\right.$ $=250 \mathrm{~K}$ ) under different surface wetting conditions is the main focus of the present study. To do so, two types of surface wetting condition have been considered based on the wettability of surface; namely hydrophilic $\left(\varepsilon_{\text {liquid-solid }} / \varepsilon_{\text {liquid-liquid }}=2.0\right)$, and hydrophobic $\left(\varepsilon_{\text {liquid- }}\right.$ ${ }_{\text {solid }} / \varepsilon_{\text {liquid-liquid }}=0.5$ ) for three different solid substrate materials such as platinum, silver and aluminum.

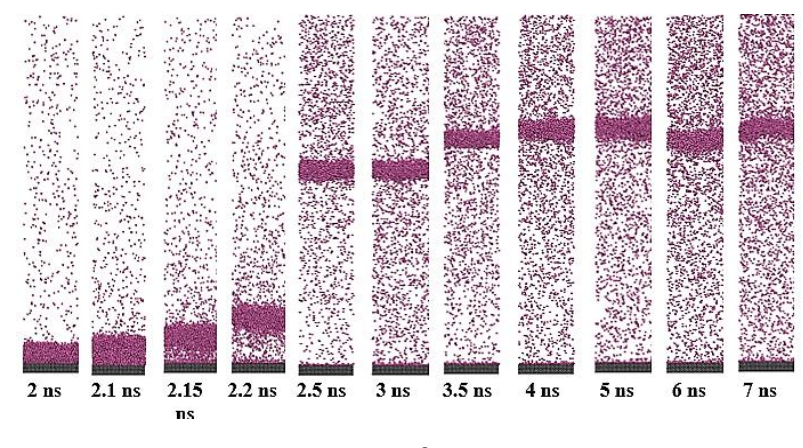

Pt-surface
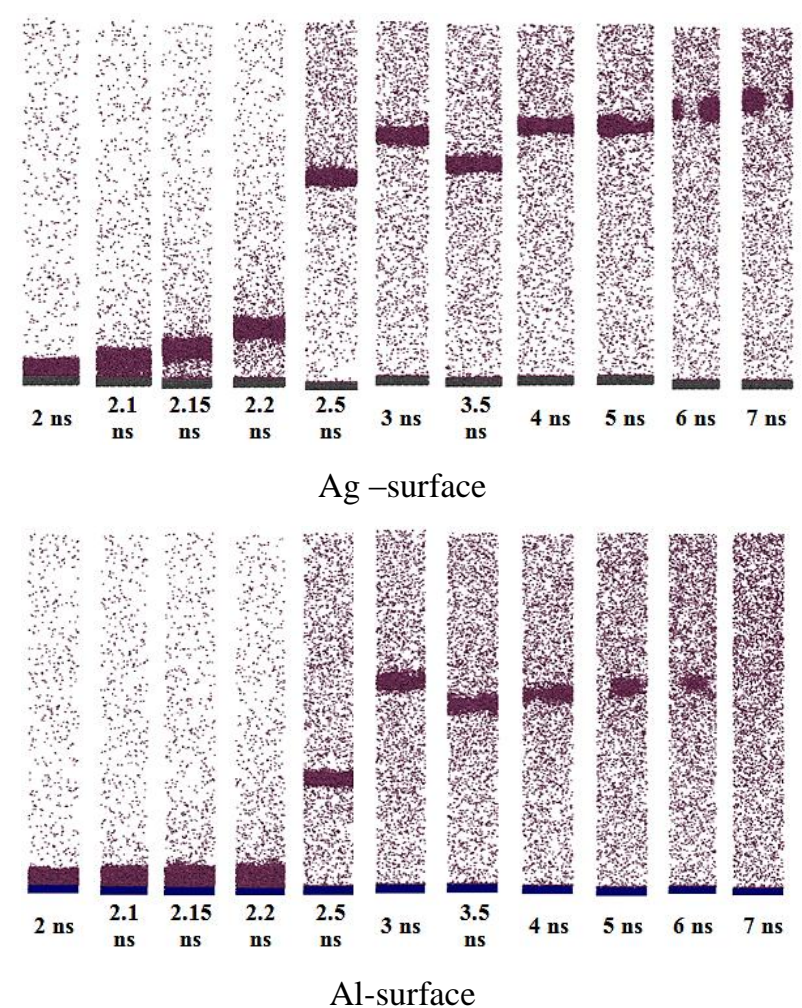

Fig. 3.Snapshots from the simulation domain for, hydrophilic Pt (top), Ag (middle) and Al (bottom) surface. 
The snapshots of simulation domain during nonequilibrium simulation period with $\mathrm{Pt}, \mathrm{Al}$ and $\mathrm{Ag}$ substrate with hydrophilic and hydrophobic are shownin Figs. 3 and 4 respectively. From the snapshots, it is very clear that the mode of phase change is significantly different for hydrophilic and hydrophobic cases: the explosive boiling phenomena occurs in case of hydrophilic surface as liquid layers are lifted up like clusters of atoms while in case of hydrophobic surface these phenomena explosive boiling does not occur and rather argon atoms leave the liquid layers as individual atoms representing a diffusion controlled phase change process which has been termed as "normal boiling/evaporation" in the contemporary literature.

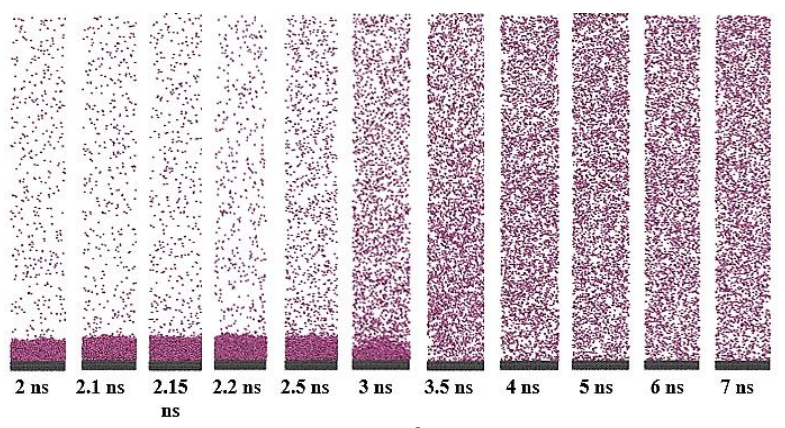

Pt-surface

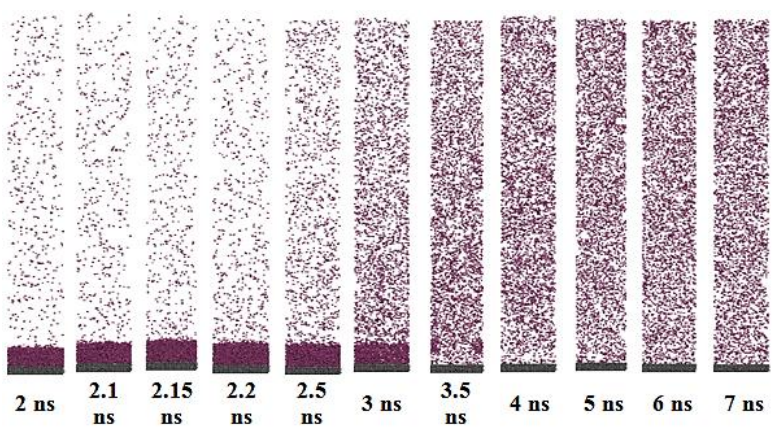

Ag-surface

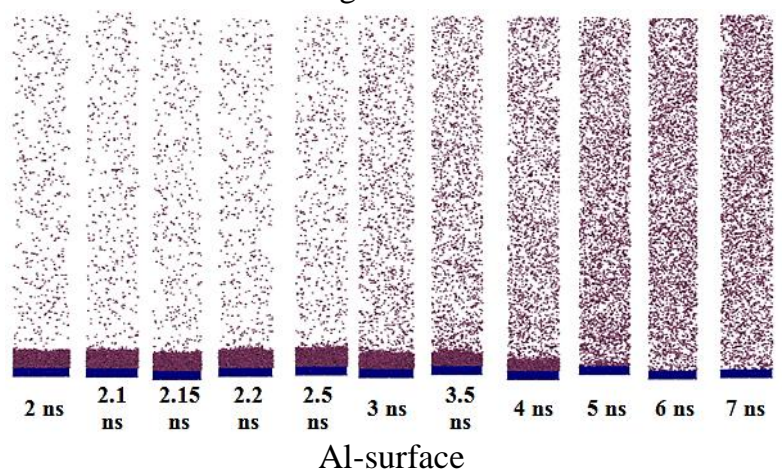

Fig. 4. Snapshots from the simulation domain for, hydrophobic Pt (top), Ag (middle) and $\mathrm{Al}$ (bottom) surface.

For hydrophilic surface as shown in Fig. 3, as the temperature of the solid wall suddenly increased from $90 \mathrm{~K}$ to $250 \mathrm{~K}$, liquid argon changes phase rapidly i.e. explosive boiling occurs; liquid layer adjacent to solid wall exceeds the critical temperature and instantly vaporizes while other layers above are still in the liquid phase. The pressure of this vaporized layer pushes the liquid above it and therefore, liquid layers separate from the solid wall as a large liquid cluster. From the snapshots, it is difficult to find out for which surface material the explosive boiling phenomena occurs quickly but a closer look suggests that it occurs first in case of Ag-surface, then for Pt-surface and last for Alsurface. From the number density profile of argon which is discussed later in this section this fact is also verified.

In case of hydrophobic surface as shown in Fig. 4, the explosive boiling phenomena does not occur and argon atoms enter the vapor region as individual atom like the low temperature case or evaporation occurs but in higher rate.

The temperature history of the system for different cases is shown in Fig. 5. For hydrophilic surface, initially the temperature of the liquid increases very sharply, but after some time it starts decreasing following the separation of the liquid argon layer from the solid wall. As the temperature of the solid wall is increased very rapidly from $90 \mathrm{~K}$ to $250 \mathrm{~K}$, the liquid layer adjacent to the solid wall exceed the critical temperature and instantly vaporize while other layers above are still in the liquid phase. The thrust of this vaporized layer pushes the liquid above it and therefore liquid layers separate from the solid wall. The energy flow from the solid wall to the liquid is hindered by the low density vapor region adjacent to the solid wall therefore the temperature of the liquid region falls temporarily.

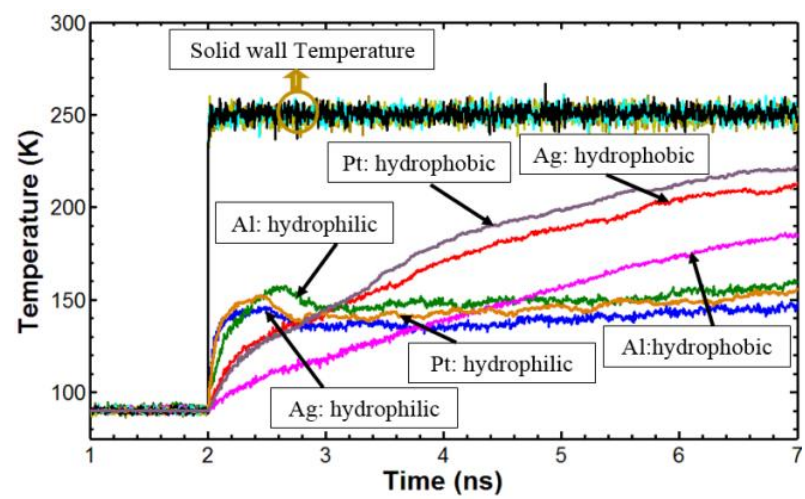

Fig. 5.Temperature history of argon and solid wall.

As expected, for hydrophilic case, the quicker the occurrence of explosive boiling, the lower is the temperature of liquid argon because, if the liquid layer is detached from the solid surface quickly by the adjacent vapor layer, then less amount of energy will be transferred to the argon atoms located above. For hydrophobic surface, the temperature profiles follows the same trend as the low temperature or evaporation case, i.e. with time temperature of argon increases gradually and approaches the solid wall temperature of $250 \mathrm{~K}$. For this case, the argon attains highest temperature for Pt-surface while the lowest temperature is obtained for Al-surface. 
Figure 6 shows the pressure history of the system for all the cases. Like the low temperature case, as the volume of the system is constraint, the pressure profiles for all the cases exactly follows the same trend of temperature profiles.

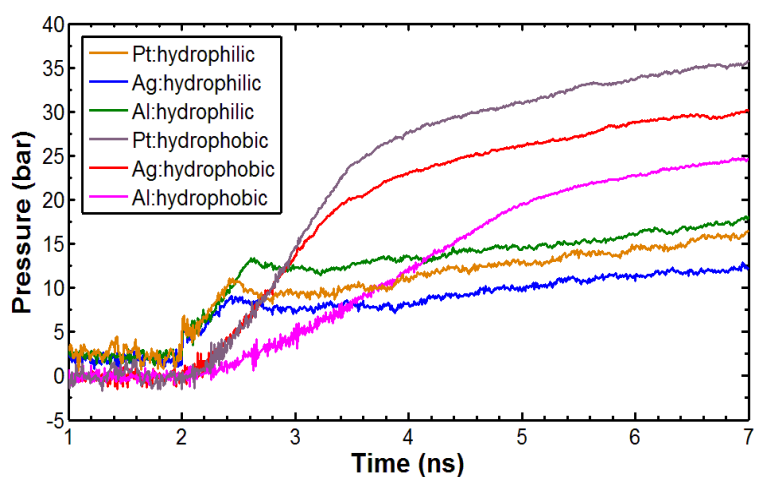

Fig. 6. Pressure history of the simulation domain.

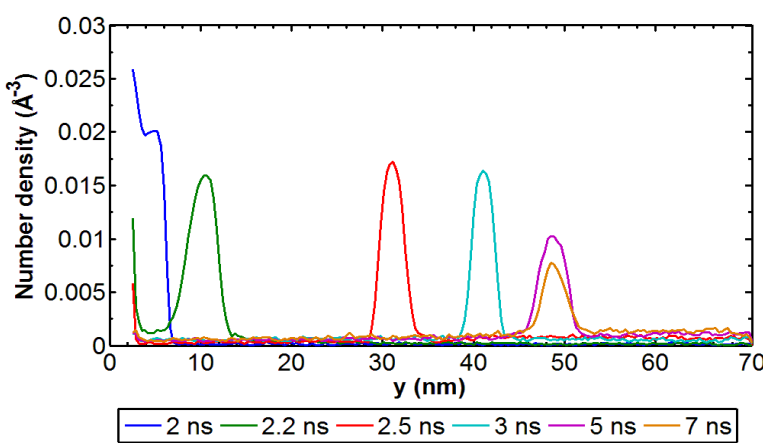

Fig. 7. Number density profile of argon on hydrophilic Pt-surface.

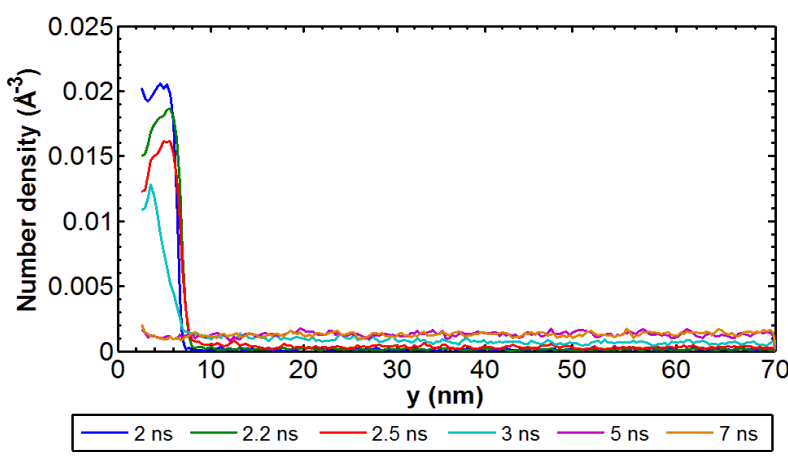

Fig. 8. Number density profile of argon on hydrophobic Pt-surface.

Figure 7 show a representative variation of number density profile of argon for hydrophilic Pt-surface along the height of the simulation domain for different instances of time. As shown in Fig. 3, explosive boiling occurs for this particular case and thus as time progresses a cluster of liquid moves away from the solid surface as shown in Fig. 7.The region of high density peaks appearing in the curve show the instantaneous locations of liquid argon cluster at different times. As shown in Fig. 7, a cluster of liquid starts to move away from the solid surface at time, $t=$
$2.2 \mathrm{~ns}$, and moves upward with time. The height of the density peaks decreases with time as argon atoms disperse from the liquid cluster to the vapor region. Figure 8 shows temporal number density profiles of argon for hydrophobic Pt-surface. From Fig. 8, it is evident that no liquid cluster is formed like the hydrophilic case rather liquid argon atoms moves away from the solid surface as individual atoms just like normal evaporation. Snapshots of Fig. 4 suggests that, the density of argon atoms becomes uniform after $3.5 \mathrm{~ns}$ for Pt-surface, $4 \mathrm{~ns}$ for Ag-surface and $5 \mathrm{~ns}$ for Alsurface. Figure 7 shows that, at $\mathrm{t}=5 \mathrm{~ns}$ and $7 \mathrm{~ns}$, density of argon has become uniform indicating that the atomic distribution achieves some sort of equilibrium conditions.

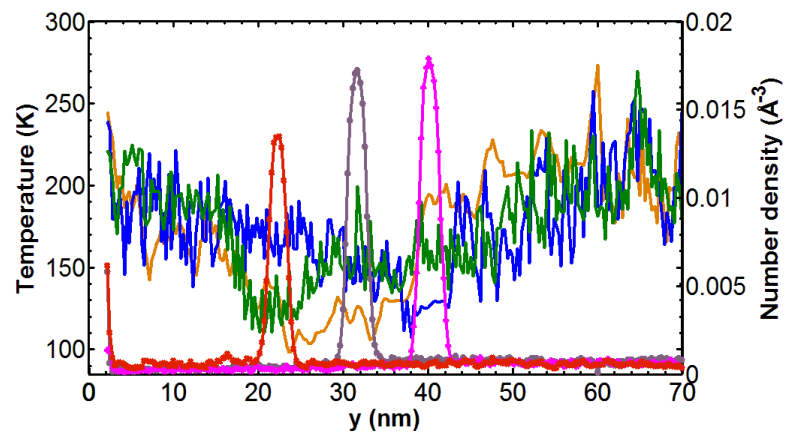

Pt:temp. -Ag:temp. $\longrightarrow$ Al:temp. $\multimap$ Pt:density $\longrightarrow$ Ag:density $\longrightarrow$ Al:density

Fig. 9. Spatial distribution of temperature and density of argon for hydrophilic $\mathrm{Pt}, \mathrm{Ag}$, and Al-surface at $2.5 \mathrm{~ns}$.

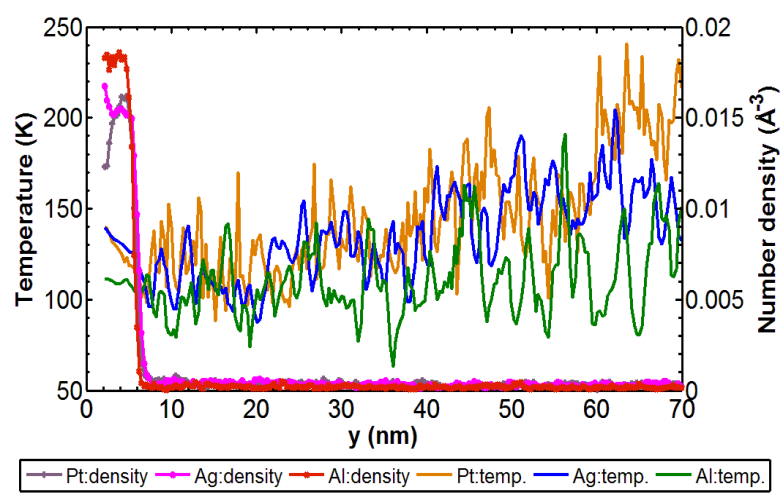

Fig.10. Spatial distribution of temperature and density of argon for hydrophobic Pt, Ag and Al-surface at 2.5ns.

The spatial distribution of temperature and number density of argon hydrophilic and hydrophobic Pt, Ag and Al-surface for a particular time instant at $\mathrm{t}=2.5 \mathrm{~ns}$, are shown in Figs. 9 and 10 respectively. As shown in Fig. 9, the liquid cluster that has been splashed away up from the wall due to heating, travel differential distances depending of the substrate material. For example: the cluster location is at a height of 20-25 nm, 30-35 nm and 38-43 nm height for $\mathrm{Al}, \mathrm{Pt}$ and $\mathrm{Ag}$ substrate respectively. The spatial temperature distributions for these three cases as shown in Fig. 9, point out that the temperature of argon at the location of 
"liquid cluster" is below the critical temperature (150 $\mathrm{K})$ that is a thermodynamic proof of the fact that the cluster is in liquid form. Below the liquid cluster region the temperature of argon atoms are higher than the critical temperature which suggests that they are in vapor phase. For the hydrophobic case phase change occurs in the form of diffusive evaporation unlike the hydrophilic case. As depicted in Fig. 10, at time, $t=2.5$ ns, significant number of liquid atoms remain adjacent to the surface.

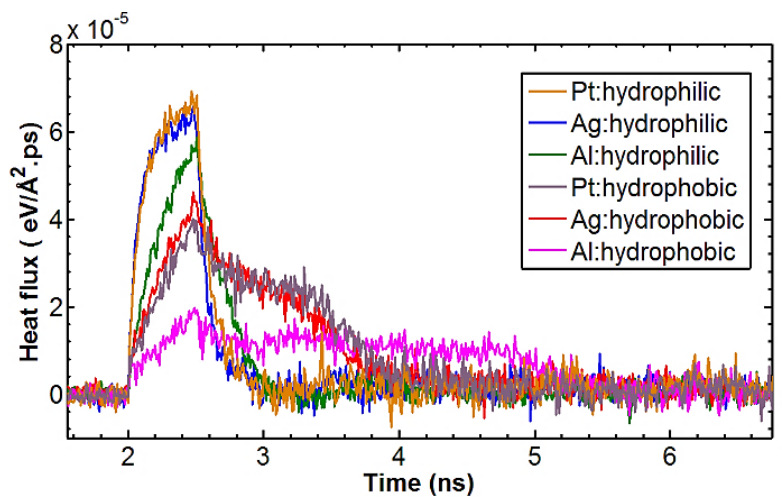

Fig. 11.Temporal variation of wall heat flux.

Figure 11 depicts the heat flux normal to the solid wall for both hydrophilic and hydrophobic cases of all three materials under consideration. For hydrophilic case, at the moment when the explosive boiling starts to take place, a huge amount of heat is transferred to the liquid adjacent to solid and after that there is a sudden drop of heat flux as indicated in Fig. 11. For hydrophobic case, similar profiles are observed but with smaller peaks and heat flux decreases much slowly. As obtained in the present study, the value of heat flux is higher in case of hydrophilic surface and maximum in case of Pt-surface, which is around $7 \times 10^{-5} \mathrm{eV} / \mathrm{A}^{2} \mathrm{ps}$ or $1120 \mathrm{MW} / \mathrm{m}^{2}$. For hydrophobic surface, the maximum magnitude of heat flux is around $4.2 \times 10^{-5} \mathrm{eV} / \mathrm{A}^{2} \mathrm{ps}$ or $672 \mathrm{MW} / \mathrm{m}^{2}$ for $\mathrm{Ag}$ surface. As shown in the snapshots of Fig. 3, in case of hydrophilic case, explosive boiling occurred in case of $\mathrm{Pt}, \mathrm{Ag}$ and $\mathrm{Al}$ surface. From Fig. 11, it is observed that, when explosive boiling occurs the magnitude of heat flux increases considerably and the value reaches one order of magnitude higher than the theoretical maximum value of heat flux, $q_{\max , \max }$ as proposed by Gambill and Lienhard [13]. The profiles of heat flux of Fig. 11 shows excellent agreement with the study performed by the Yamamoto and Matsumoto [14].

Heat Flux profiles from Fig. 11 also confirms that the hydrophilic surface transfers energy more effectively. Heat flux for explosive boiling for both hydrophilic and hydrophobic cases is much larger compared to the result of Fig. 11. This anomaly occurs because, a very high heat flux is suddenly added within a very short time when explosive boiling begins and thus the energy profile consists a large jump of magnitude of energy in this region and when the heat flux is calculated, it fails to capture the true nature of the phenomena.
FollowingFig. 11, the magnitudes of heat flux during explosive boiling which occurs in case of hydrophilic surface found to be one order of magnitude higher than the theoretical maximum of heat flux, $q_{\max , \max }$ as defined by Gambill and Lienhard [13], but for hydrophobic case the magnitudes of the heat flux are in the order of $q_{\max , \max }$. In hydrophobic case, as shown in the snapshots of Fig. 4, explosive boiling did not occur and instead normal boiling or evaporation took place. Therefore, heat flux profiles of Fig. 11 show that, for hydrophilic surface the rate of decrease of heat flux is very rapid and within $3 \mathrm{~ns}$ the magnitude of heat flux reaches to zero due to the occurrence of explosive boiling phenomena. But for hydrophobic surface, as evaporation takes place for relatively longer time period and thus heat flux profiles assume higher values for longer time period in comparison to its hydrophilic counterpart as depicted in Fig. 11.

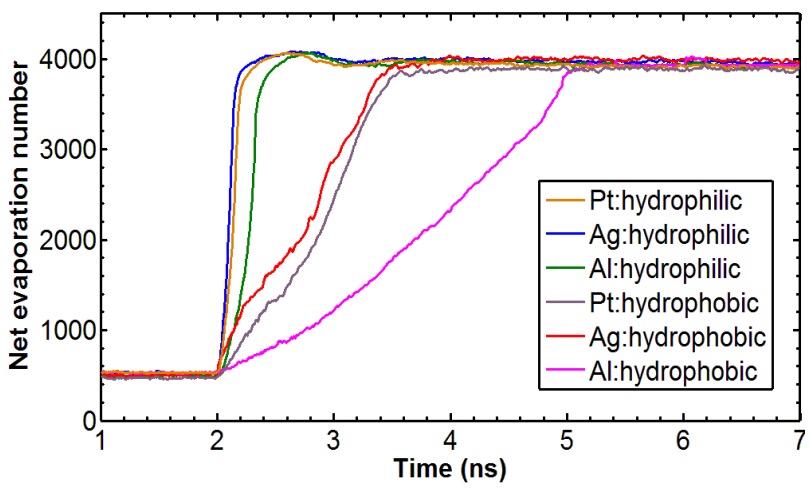

Fig.12. Time variation of net evaporation number

Figure 12 shows the net evaporation number for high temperature case. As expected, the number of argon atoms in vapor region remains constant during equilibrium period. When the temperature of the solid wall is increased from $90 \mathrm{~K}$ to $250 \mathrm{~K}$, for hydrophilic surface explosive boiling takes place and as soon as the liquid cluster moves away from the solid surface, the number of argon atoms increases almost instantaneously to a higher value. From Fig. 12, it is clear that, for hydrophilic surface the explosive boiling in case Ag-surface and Pt-surface take almost at same instance of time while in case of Al-surface, it happens lately in comparison to Ag-surface and Pt-surface. For hydrophobic surfaces, evaporation in the form of diffusion takes place, therefore the number of atoms in vapor region increases gradually untill atomic distribution achieves equilibrium state. For the hydrophobic case, the rate of evaporation follows the sequence: highest in case of Ag-surface and lowest in case of Al-surface. The number of atoms for all surface materials after $7 \mathrm{~ns}$ is almost same. The atomic distribution as depicted in Fig. 3(b) are in agreement with the finding of Fig. 12 that is evaporation stop about $\mathrm{t}=3.5 \mathrm{~ns}$ for hydrophobic Pt and Ag surfaces while for hydrophobic Al surface it ceases at $t=5 \mathrm{~ns}$. 


\section{Conclusion}

Using non-equilibrium molecular dynamics (NEMD) simulation the phase change phenomena for a thin liquid argon film over a solid substrate at different surface wetting conditions (hydrophilic and hydrophobic)have been studied for different substrate materials (platinum, silver and Aluminium) at high degree of wall superheat.The heat transfer rate from substrate to the liquid significantly increases with the increase of solid-liquid interaction potential, i.e. with the increase of the surface wettability. Among the three materials that have been considered in this study, Aluminium was the least effective while Platinum and silver showed similar characteristics for heat and mass transfer. Explosive boiling was observed in case of hydrophilic surface, but for hydrophobic surfaces, diffusive evaporation was observed. From heat flux point of view, the transfer of heat in case of hydrophilic surface was much higher than the hydrophobic surfaces.

\section{References}

[1] T. Kimura, S. Maruyama, Molecular dynamics simulation of heterogeneous nucleation of a liquid droplet on a solid surface, MicroscaleThermophys. Engg. 6, pp. 3-13, 2002

[2] M. Sekine, K. Yasuokaa,, T. Kinjob, M. Matsumoto, Liquid-vapor nucleation simulation of Lennard-Jones fluid by molecular dynamics method, Fluid Dyn. Research 40, pp. 597 - 605, 2008.

[3] J. Yu, H. Wang, A molecular dynamics investigation on evaporation of thin liquid films, Int. J. Heat Mass Transfer 55, pp. 1218-1225, 2012.

[4] G. Nagayama, S. Shi-iki, T. Tsuruta, Effects of naostructures on surface wettability: a molecular dynamics study, Trans. Japan Society of Mechanical Engg. B 73 (728), pp.1084 - 1091, 2007.

[5] S.C. Maroo, J.N. Chung, Molecular dynamic simulation of platinum heater and associated nanoscale liquid argon film evaporation and colloidal adsorption characteristics, J. Colloid Interface Sci. 328, pp. 134-146, 2008.

[6] A.K.M.M. Morshed, Taitan C. Paul, Jamil A. Khan, Effect of nanostructures on evaporation and explosive boiling of thin liquid films: a molecular dynamics study, Applied Physics A 105, pp. 445$451,2011$.

\section{Nomenclature}

$\begin{array}{ll}\mathrm{P} & \text { - Pressure [bar] } \\ \mathrm{q}_{\max , \max } & \text {-Theoretical maximum value of heat } \\ \mathrm{r} & \text { flux }[\mathrm{W} / \mathrm{m} 2] \\ \mathrm{t} & \text { - Inter-molecular distance }[\AA] \\ \mathrm{T} & - \text { Time }[\mathrm{ns}] \\ \mathrm{X} & \text { - Temperature }[\mathrm{K}] \\ \mathrm{y} & \text { - Coordinate along } \mathrm{X} \text { axis } \\ \mathrm{Z} & \text { - Coordinate along } \mathrm{Y} \text { axis } \\ \varepsilon & \text { - Coordinate along } \mathrm{Z} \text { axis } \\ \sigma & \text { - Energy parameter }[\mathrm{eV}] \\ \varphi & \text { - Length parameter }[\AA]\end{array}$

[7] H.R. Seyf, Y. Zhang, Molecular Dynamics Simulation of Normal and Explosive Boiling on Nanostructured Surface, ASME J. Heat Transfer 213 (135) 121503, pp. 1-8, (2013)

[8] S. Maruyama, T. Kimura, A molecular dynamics simulation of a bubble nucleation on solid surface, Int. J. Heat Technol. 18, pp. 69-74, 2000.

[9] A. Hens, R. Agarwal, G. Biswas, Nanoscale study of boiling and evaporation in a liquid Ar film on a Pt heater using molecular dynamics simulation, Int. J. Heat Mass Transfer 71, pp. 303-312, 2014.

[10]J.E. Lennard-Jones, A.F. Devonshire, Philos. Trans. R. Soc. Lond. Ser. A, Math. Phys. Sci. 163, pp. 53-70, 1937.

[11]LAMMPS User's Manual (http://lammps.sandia. gov - Sandia National Laboratories, USA)

[12] Humphrey, W., Dalke, A., and Schulten, K., "VMD: Visual Molecular Dynamics," Journal of Computational Physics, 117(1), pp. 1-19,1996.

[13] W.R. Gambill, and J.H. Lienhard, "An Upper Bound for the Critical Boiling Heat Flux," ASME Journal of Heat Transfer, 111(3), PP. 53-70,1989.

[14] T. Yamamoto, M. Matsumoto, Initial stage of nucleate boiling: molecular dynamics investigation, J. Therm. Sci. Technol. Jpn. 7, pp. 334-349, 2012. 